\title{
Variable Porosity of the Pipeline Embolization Device in Straight and Curved Vessels: A Guide for Optimal Deployment Strategy
}

\author{
M. Shapiro, E. Raz, T. Becske, and P.K. Nelson
}

\begin{abstract}
BACKGROUND AND PURPOSE: Low-porosity endoluminal devices for the treatment of intracranial aneurysms, also known as flow diverters, have been in experimental and clinical use for close to 10 years. Despite rigorous evidence of their safety and efficacy in well-controlled trials, a number of key factors concerning their use remain poorly defined. Among these, none has received more attention to date than the debate on how many devices are optimally required to achieve a safe, effective, and economical outcome. Additional, related questions concern device sizing relative to the parent artery and optimal method of deployment of the devices. While some or all of these issues may be ultimately answered on an empiric basis via subgroup analysis of growing treatment cohorts, we believe that careful in vitro examination of relevant device properties can also help guide its in vivo use.
\end{abstract}

MATERIALS AND METHODS: We conducted a number of benchtop experiments to investigate the varied porosity of Pipeline Embolization Devices deployed in a simulated range of parent vessel diameters and applied these results toward conceptualizing optimal treatment strategies of fusiform and wide-neck aneurysms.

RESULTS: The results of our studies confirm a predictable parabolic variability in device porosity based on the respective comparative sizes of the device and recipient artery, as well as device curvature. Even modest oversizing leads to a significant increase in porosity.

CONCLUSIONS: The experiments demonstrate various deleterious effects of device oversizing relative to the parent artery and provide strategies for addressing size mismatches when they are unavoidable.

ABBREVIATIONS: PED = Pipeline Embolization Device; PUFS = Pipeline for Uncoilable and Failed Aneurysms; TZ = transition zone

$\mathbf{T}$ he clinical experience with the Pipeline Embolization Device (PED; Covidien, Irvine, California) is characterized by heterogeneity in the selection of device size, number, and deployment technique. For example, the average number of devices used in the Pipeline Embolization Device for the Intracranial Treatment of Aneurysms Trial (PITA) ${ }^{1}$ and Pipeline for Uncoilable and Failed Aneurysms (PUFS) ${ }^{2}$ trials is 1.52 and 3.1, respectively, a notable difference notwithstanding the larger dimensions of PUFS aneurysms. The "less is more" model remains predominant in Europe and South America, whereas a more varied mixture of strategies

Received April 25, 2013; accepted after revision August 3.

From the Departments of Radiology, Bernard and Irene Schwartz Neurointerventional Radiology Section, (M.S., E.R., T.B., P.K.N.), Neurosurgery (P.K.N.), and Neurology (M.S., T.B.), New York University Langone Medical Center, New York, New York.

Pipeline devices used for this research were donated by Covidien. No funds were solicited or provided for this study from any source.

Please address correspondence to Maksim Shapiro, MD, Departments of Radiology and Neurology, Bernard and Irene Schwartz Neurointerventional Radiology Section, NYU Langone Medical Center, 660 First Ave, 7th floor, New York, NY 10016; e-mail: maksim.shapiro@nyumc.org, neuroangio@neuroangio.org

Indicates article with supplemental on-line figures.

http://dx.doi.org/10.3174/ajnr.A3742 seems to exist in North America and Australia. Neither approach has been subjected to rigorous targeted investigation, though one might argue that the results of the best-controlled study to date-PUFSset the metrics of efficacy based on using multiple-coverage constructs, thereby placing the burden of proof on the minimalist approach to demonstrate superiority or equipoise.

An issue that necessarily arises when considering the use of a single PED in the treatment of a complex-neck aneurysm concerns the mechanical behavior of the braided device when forced to accommodate significant mismatch in the diameters of the stented vascular segment. In a significant number of very wide-neck or fusiform aneurysms affecting the segments of the internal carotid artery for which the device is indicated, the proximal and distal landing zones tend to be of different diameters so that placement of a single device necessarily requires oversizing at one (usually the distal) end of the recipient vessel. What consequences this strategy may have on treatment efficacy may still be considered unclear, though a growing body ${ }^{3}$ of benchtop, ${ }^{4,5}$ flow dynamics, ${ }^{6}$ and animal literature ${ }^{7}$ suggests that deliberate or inadvertent oversizing of the device is likely to be detrimental to the intended flow modification. 

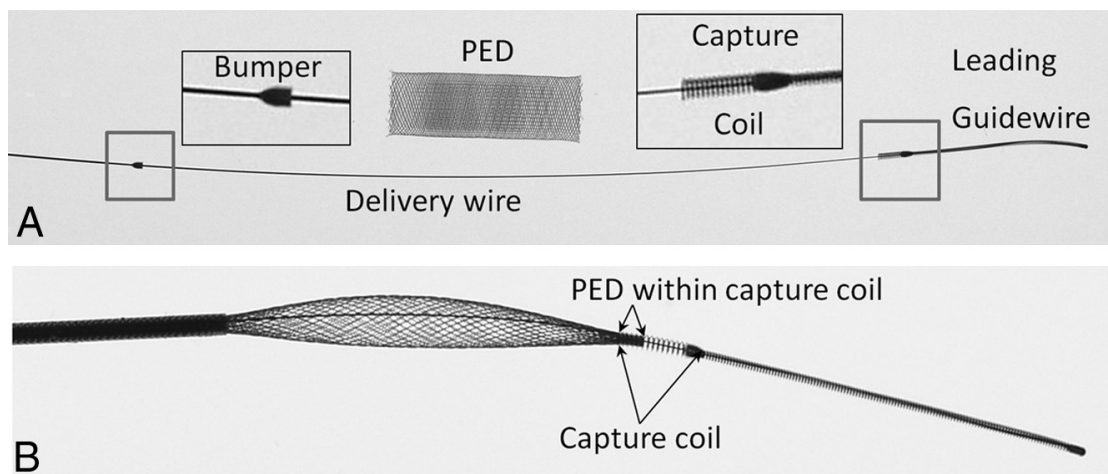

FIG 1. A, Pipeline device delivery system components. B, Partially unsheathed device demonstrating the segment inserted into the capture coil.
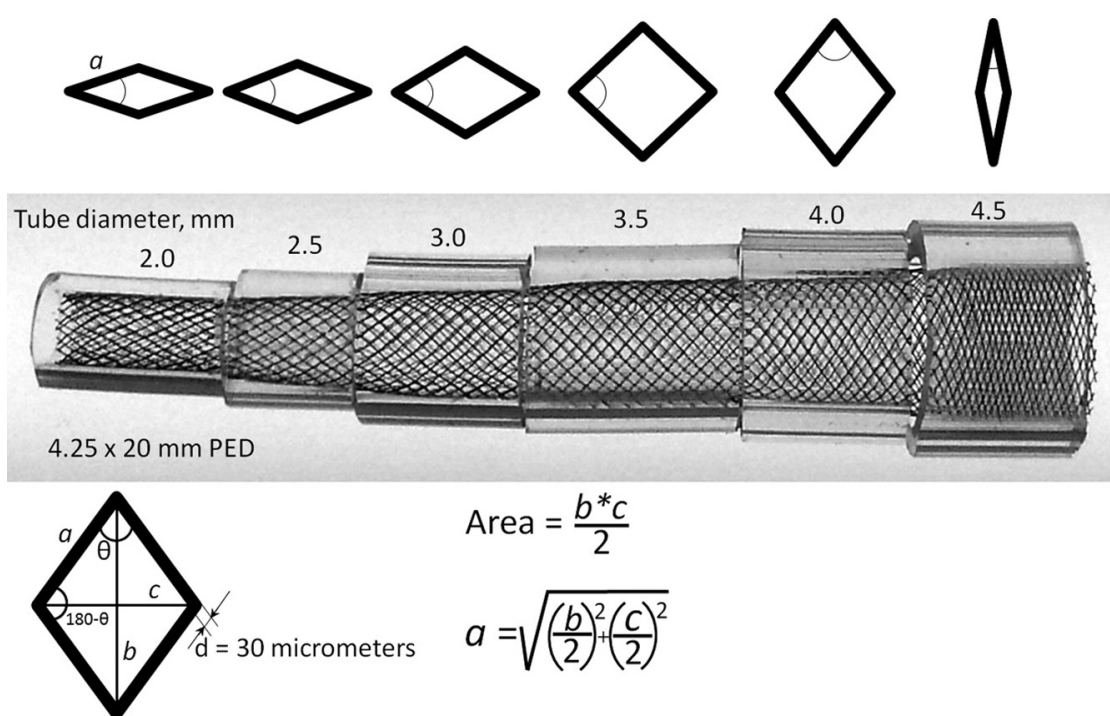

$$
\begin{aligned}
& \text { Area }=\frac{b^{*} c}{2} \\
& a=\sqrt{\left(\frac{b}{2}\right)^{2}+\left(\frac{c}{2}\right)^{2}}
\end{aligned}
$$

FIG 2. Representative sample of an experimental setup, with a $4.25 \times 20 \mathrm{~mm}$ device inserted into plastic tubes of $0.5-\mathrm{mm}$ incremental diameters. The corresponding parameters of cell length $a$ and angle $\theta$ are shown, demonstrating that $a$ remains relatively constant so that porosity is determined primarily by variance in $\theta$.

A related issue concerns device behavior along vessel curvatures, where the degree of metal coverage is found to be inhomogeneous, predictably varying from the highest coverage (lowest porosities) along the inner curve and decreasing gradually to the minimum coverage seen on the outer curvature. The extent to which this increases porosity under different circumstances has been investigated for some devices ${ }^{3,5,8}$ but is not established for the PED.

\section{Device Construction}

The PED is a self-expanding, cylindric, braided device consisting of 48 strands of cobalt-chromium and platinum-tungsten wire, in a 3:1 respective ratio, wound by a braiding machine to produce devices ranging from 2.5 to $5.0 \mathrm{~mm}$ in nominal diameter, with lengths varying from 10 to $35 \mathrm{~mm}$. The device is mounted within the delivery sheath by stretching, and its leading edge is packaged beneath a capture coil to protect the strands during advancement within the microcatheter (Fig 1). During delivery, the device may expand to its maximum size, which is $0.25 \mathrm{~mm}$ larger than nominal diameter when unconstrained across the aneurysm neck, or it may conform to the diameter of the vessel in which it is implanted. For the subsequent discussion, the term "coverage" will be used interchangeably with "metal coverage," defined as the percentage of artery surface area covered with the metal strands of the device, reflecting the inverse of the term "porosity," which here simplistically refers to the percentage of uncovered artery area.

On close inspection, the ultrastructure of the device consists of a series of curved rhomboid cells. (Fig 2). The angle $\theta$, or pitch, of the strands at nominal size is set during manufacture, along with the diameter of the strand (average: $30 \mu \mathrm{m}$ per manufacturer's specifications) and number of strands, and determines the porosity and pore (cell) size of the device. However, when a device is placed into vessels of progressively smaller sizes relative to its nominal diameter, the pitch angle of the cells changes proportional to the degree of device constraint, providing lower coverage and increased surface porosity until a maximum porosity is reached at the lowest metallic coverage. This minimum coverage results when the cell angle $\theta$, for a given cell side length, reaches $90^{\circ}$, corresponding to a square configuration. With higher degrees of constraint (even more oversizing), the cells again assume a diamond shape, oriented now along the long axis of the device, thereby again decreasing overall porosity (Fig 2) and increasing the surface area coverage. Thus, the theoretic curve of coverage versus vessel diameter for each device has a parabolic shape as long as the cell length, $a$, remains constant, and this turns out to be essentially true. Please note that this relationship is different from the "ideal" rhombus, whose area is independent of $\theta$, whereas rhombi defined by braids of defined thickness $(30 \mu \mathrm{m})$ display a more complex angle-area relationship. Under conditions of curvature, however, both the cell angle $\theta$ and the side length $a$ vary so that the range of porosities throughout a cross-section of the parent artery along a curve may become quite substantial.

\section{MATERIALS AND METHODS}

A number of Pipeline Embolization Devices were deployed within clear plastic tubes of predefined diameters ranging from 2.0 to 5.0 $\mathrm{mm}$, in increments of $0.5 \mathrm{~mm}$. The constructs and an adjacent calibration ruler were then photographed at close range with 2 conventional cameras (Fig 2). Repeated measurements of the long, $b$, and short, $c$, cell axes were made for adjacent cells of each segment, and measurements were averaged to produce values and 
Pipeline device metal coverage \pm SD for each tested device diameter and recipient tube diameter

\begin{tabular}{ccccc}
\hline \multirow{2}{*}{$\begin{array}{c}\text { Tube Diameter } \\
(\mathbf{m m})\end{array}$} & \multicolumn{4}{c}{ Nominal Device Diameter (mm) } \\
\cline { 2 - 5 } & $\mathbf{4 . 7 5}$ & $\mathbf{4 . 2 5}$ & $\mathbf{3 . 7 5}$ & $\mathbf{3 . 2 5}$ \\
\hline 5.0 & $27 \pm 4 \%$ & & & \\
4.5 & $20 \pm 3 \%$ & $36 \pm 5 \%$ & & \\
4.0 & $18 \pm 6 \%$ & $21 \pm 5 \%$ & $28 \pm 7 \%$ & \\
3.5 & $18 \pm 4 \%$ & $20 \pm 5 \%$ & $22 \pm 5 \%$ & $33 \pm 4 \%$ \\
3.0 & $18 \pm 3 \%$ & $20 \pm 6 \%$ & $22 \pm 9 \%$ & $29 \pm 6 \%$ \\
2.5 & $20 \pm 4 \%$ & $22 \pm 4 \%$ & $22 \pm 6 \%$ & $26 \pm 4 \%$ \\
2.0 & $25 \pm 8 \%$ & $26 \pm 4 \%$ & $25 \pm 6 \%$ & $28 \pm 5 \%$ \\
\hline
\end{tabular}

SDs for each device and recipient tube size using open-source image-processing software, ImageJ (National Institutes of Health, Bethesda, Maryland). The average diameter of the device strands was taken to be $30 \mu \mathrm{m}$, as specified by the manufacturer. On the basis of these parameters, the cell surface area, cell angle $\theta$, cell side length $a$, and the percentage of metal coverage were calculated, with respective SD values, by using basic geometric and errorpropagation formulas.

The effects of device curvature on porosity at various sections of a $180^{\circ}$ curve were investigated by measurement of the cell area and metal coverage by using the aforementioned techniques for a $3.25 \times 20 \mathrm{~mm}$ PED. An unconstrained device configuration (maximal opening) was chosen to reflect the clinically relevant scenario of placing the device into a fusiform aneurysm arising from a curved parent vessel.

On the basis of observations of device behavior during these experiments, additional constructs were made to investigate device properties under conditions of oversizing and to demonstrate strategies for minimizing its effects.

\section{RESULTS}

\section{Device Sizing Relative to the Parent "Artery"}

The geometric metal coverage was calculated for each device within its diameter range, as shown in the Table. The cell surface area and percentage of metal coverage were found to exhibit a parabolic relationship with respect to recipient "artery" diameter, as illustrated in On-line Figs 1 and 2. Maximum coverage was observed under conditions allowing maximal device expansion, which corresponds to $0.25 \mathrm{~mm}$ above the nominal diameter. As the device is placed in proportionately smaller diameter tubes, the rhomboid cells "open" until minimum coverage is achieved at the "square" configuration, following which coverage begins to increase again (Fig 2 and On-line Fig 3). Metal coverage falls rapidly with increasing device oversizing, with minimum coverage already observed when the "artery" is only $1 \mathrm{~mm}$ smaller than nominal device diameter (Table, On-line Fig 1). Near minimal coverage is maintained for the next $\sim 1 \mathrm{~mm}$ of oversizing. For example, the coverage provided by a $4.75-\mathrm{mm}$ device is already substantially diminished when placed into a $3.75-\mathrm{mm}$ vessel and remains at near-minimum values down to $2.75 \mathrm{~mm}$. Thus, even relatively modest degrees of oversizing translate into substantially lower metallic coverage. Although the device strands are not welded to each other, the side lengths, $a$, of the rhomboid cells remain relatively constant in straight vascular segments for each device throughout its range of recipient artery diameters (On-line Fig 4) so that observed changes in metal coverage result primarily from adaptations in the cell angle, $\theta$ (On-line Fig 3). Under conditions of significant vascular/device curvature, however, both parameters change substantially, as illustrated below.

\section{Effects of Oversizing}

When deployed across a fusiform aneurysmal dilation, the effects of oversizing additionally lead to the development of a funnelshaped transitional zone (TZ) between the constrained segment of the device implanted in the recipient artery and the unconstrained segment extending across the aneurysm, particularly distally where the degree of mismatch between the device diameter and the diameter of the recipient artery is greatest (Fig 3). The dimensions of this zone of higher porosity (relative to the compressed cell structure of the fully expanded device) are determined by the magnitude of change in the device diameter while transitioning from its constrained state (implanted in the vessel beyond the aneurysm) to its unconstrained diameter (within the aneurysm) - and are fixed in that they cannot be modified (reduced) by the application of a forward load onto the freely expanded segment of the device (packing the device) (Fig 4). Therefore, oversizing will necessarily result in a segment of decreased metal coverage, which typically is located at the distal aneurysm boundary. Aside from the geometric reduction in the attenuation of metal coverage, depending on the method of deployment, this zone may contribute to the establishment of an asymmetric region of higher porosity near the aneurysm terminus, providing a zone of relatively lower resistance to inflow into the aneurysm, while the more appropriately sized proximal aneurysm segment (outflow zone) enjoys higher metal coverage, creating a mismatch in porosities across the length of the aneurysm.

A related observation was made concerning the morphology of the landing zone under conditions of device oversizing. When a device is oversized and deployed within a relatively small landing zone, the device edge tends to assume a cone-shaped morphology, a property intrinsic to all braided stent designs. This effect is exacerbated, rather than relieved, by application of forward tension (loading) onto the freely open portion of the stent (Fig 4). With progressive oversizing and increased forward load during deployment, the degree of "fishmouthing" becomes quite pronounced (Fig $4 C,-D$ ). These observations are in excellent agreement with recently published results of similar experiments by Raymond and colleagues. ${ }^{4}$ Although these extreme in vitro scenarios are not necessarily reflective of typical in vivo deployments, the combination of oversizing and short landing zones may result in suboptimal opening of the device distally, potentially favoring progressive retraction (proximal migration, "watermelon-seeding") of the unexpanded distal end of the device into the fusiform segment where the device is fully expanded. This ultimately may lead to prolapse of the distal end of the device into the aneurysm, particularly when the distalmost segment of the device is deployed under conditions of traction (stretching) or where the exiting vessel exhibits a funnel shape.

We also observed, under conditions of longer landing zones, that the effect of oversizing manifests itself as a "lip" of decreased device apposition relative to the recipient tube, which may contribute, in vivo, to development of an endoleak as the implanted portions of the device become partially overgrown by neointima (Fig 3A). The same phenomenon can also be seen in vivo (Fig 5). 

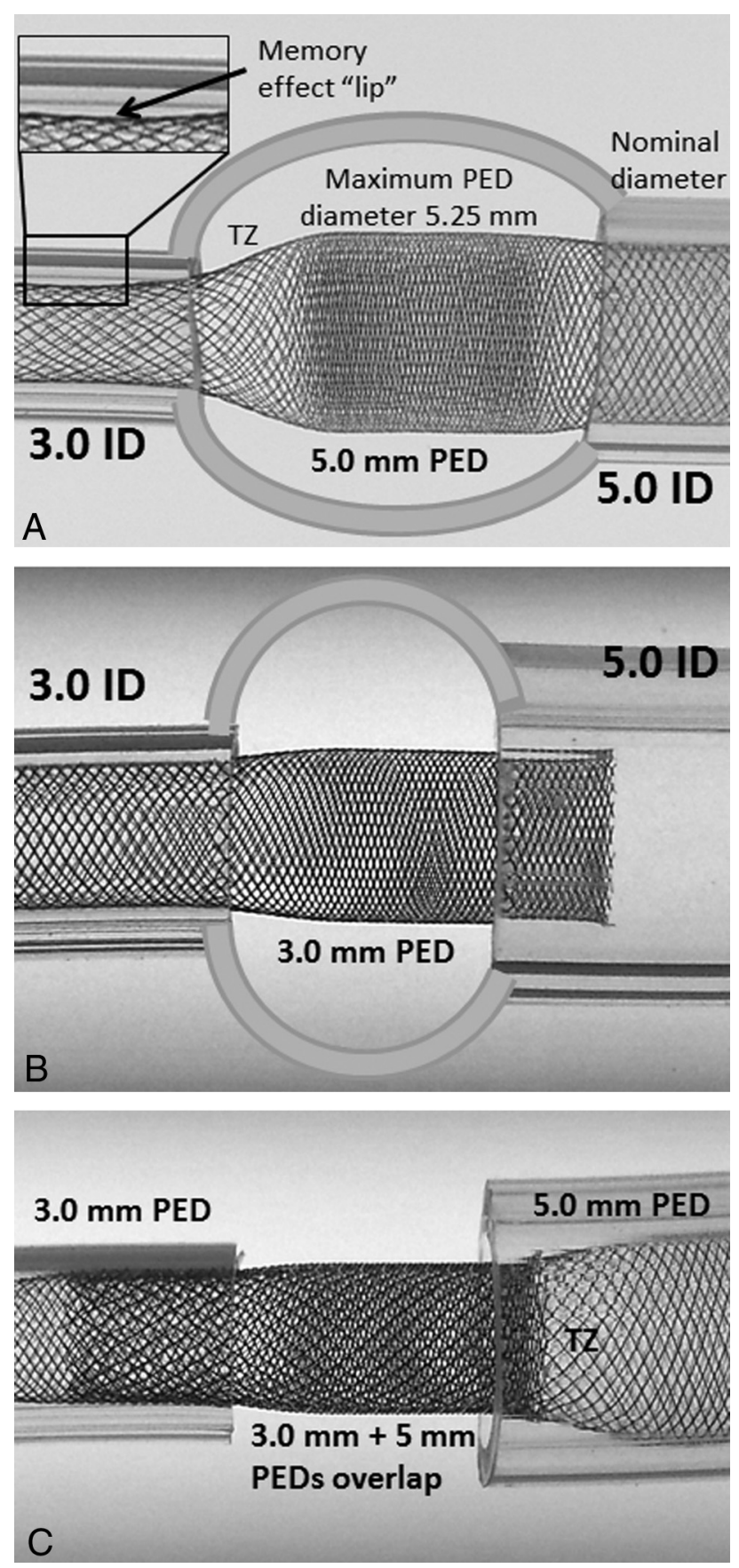

FIG 3. Morphologic effects of device oversizing and the corresponding solution. A, A model of a fusiform aneurysm with 3.0- and 5.0-mm landing zones, bridged by a single $5 \times 20 \mathrm{~mm}$ device. A transition zone of minimum coverage is created as the device is constrained from its fully opened state into the 3-mm landing zone. Despite adequate length of the "landing zone" at the 3.0-mm end, the "shape memory" of the transition zone nevertheless produces a "lip" where the device remains unapposed to the inner wall of the tube. $B$ and $C$, To address these issues, 2 devices are required, each of which is appropriately sized for its recipient artery. The first 3.0- $\mathrm{mm}$ device is deployed from the 3.0-mm-diameter vessel into the $5-\mathrm{mm}$ recipient vessel $(B)$, following which a second $5.0-\mathrm{mm}$-diameter device is telescoped into the first, with the $5.0-\mathrm{mm}$ device anchored into its $5.0-\mathrm{mm}$ vessel. Thus, the transition zone is shifted outside the aneurysm, while the aneurysmal segment receives the benefit of double coverage.
Thus, experiments of Raymond and colleagues ${ }^{9}$ and our group suggest that oversizing results in suboptimal configurations at both the distal landing zone and within the intra-aneurysmal "transition zone," which may have anatomic and physiologic implications with respect to the effectiveness of the construct.

\section{Metal Coverage along the Device Curvature}

The geometric effects of curvature on coverage are more complex than those of simple oversizing. The PED accommodates exceptionally well to high degrees of vessel curvature, in part due to the extreme flexibility of the device, which is enabled by the unfixed property of its braided filaments. When deployed along a curved vessel, the composite metal strands slide along one another, altering the dimensions, $a$, of the individual cells. This "unfixed" characteristic leads to changes in both the angle, $\theta$, and rhombus side length, $a$, resulting in the "opening" of cells along the outer curve and their progressive closure at the inner curve, in contrast to the more simple factors governing the behavior of the devices in straight segments of changing diameter, where only the angle, $\theta$, changes substantially, and the side length, $a$, remains essentially constant (On-line Fig 4). All of these accommodations take place in the unconstrained state of the device, which would correspond to its in vivo morphology under a scenario in which the devices would be used in the endoluminal treatment of a fusiform aneurysm involving a curved segment of the parent vessel. If, in addition to curvature effects, the device was also constrained within the curved parent artery, the final configuration is expected to be even more complex, with myriad possible scenarios beyond the aim of the present investigation. For the unconstrained device, the representative percentage of metal coverage along different sections of a $180^{\circ}$ curve is shown in Fig 6. From these data, it can be readily appreciated that while coverage is modestly reduced at the outer curvature, it is dramatically increased along the inner curve. While the effects of these changes on flow patterns, particularly at the outer curve, remain to be defined, there is experimental evidence to support the notion that treatment efficacy of aneurysms arising from the outer curve of a parent artery is reduced after attempted treatment with single-device placement. ${ }^{8}$

\section{DISCUSSION}

The above experiments serve to document certain features of the Pipeline Embolization Device and additionally illustrate potentially unanticipated effects on device porosity, final construct geometry, and implant stability arising from the use of oversized PEDs in the treatment of complex neck aneurysms. Other groups have made similar observations with similar endoluminal devices based on in vitro testing, flow modeling, and animal experiments. ${ }^{6,8-10}$ Circumstances in which these effects are likely to be encountered include the treatment of the following: 1) complex fusiform aneurysms; 2) large dysplastic saccular aneurysms, particularly those near-circumferentially involving the parent artery; and 3) large, broad-neck aneurysms involving locations associated with significant changes in vessel diameter proximal and distal to the aneurysm neck. Under this latter condition, treatment of the target aneurysm with a single device necessitates oversizing at the smaller diameter landing zone (because no variable-diameter devices are currently on the market). This unavoidable outcome can 

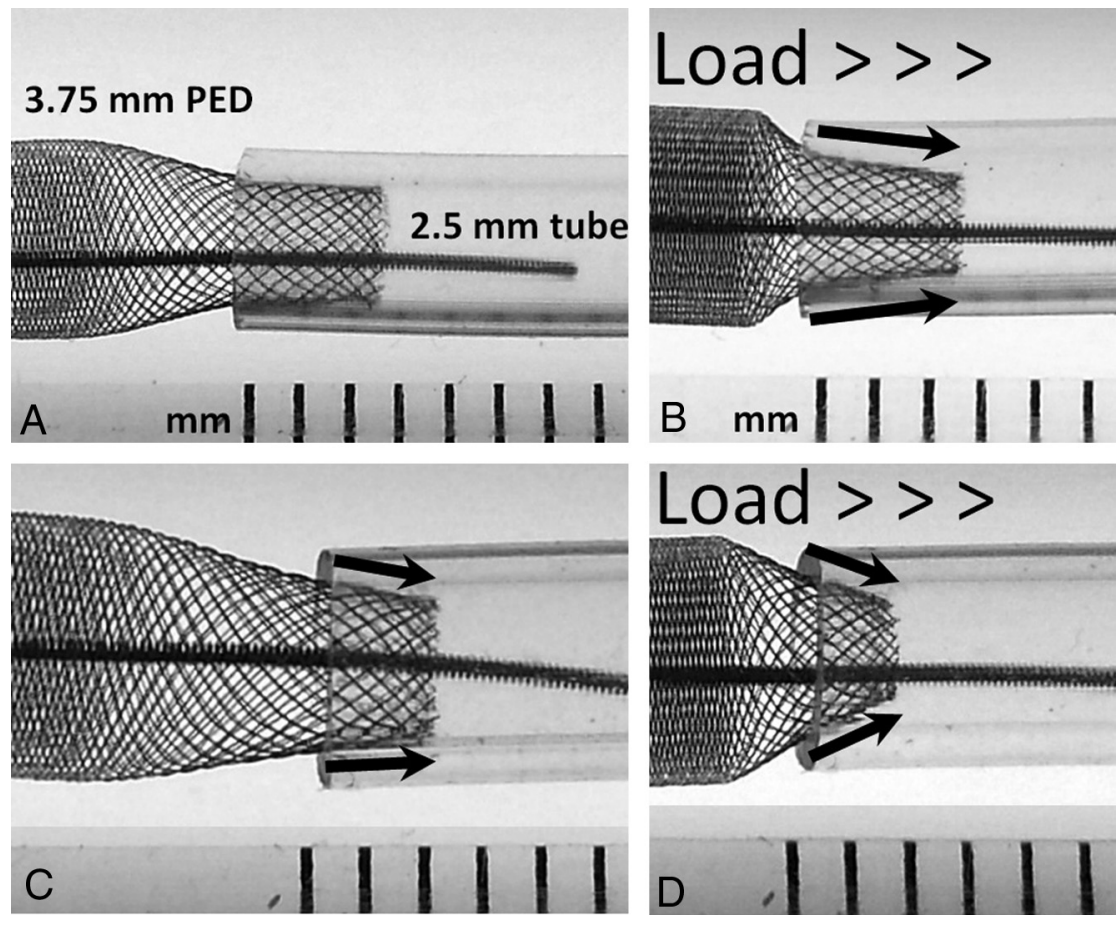

FIG 4. The "fishmouth" configuration of oversized devices in scenarios of short landing zones. A 2.5-mm landing zone $(A)$ leads to no appreciable fishmouth configuration, unless the forward load is applied to the freely expanded portion of the device, as might be done in an attempt to better seat the device into the recipient artery $(B)$. This, in fact, has the effect of decreasing device apposition to the wall, because the foreshortened area of decreased coverage remains unchanged, while a degree of fishmouthing is now present (arrows), due to an increase in the centripetal force vector along the transition zone angle. These effects are magnified when the landing zone decreases to approximately $1.5 \mathrm{~mm}$ in length $(C$ and $D)$. Altogether, these images suggest that the deleterious effects of oversizing are only likely to be exacerbated by attempts to force the device into the undersized artery.

be further exaggerated in proportion to the length of the device chosen, because the extension of longer devices proximally typically carries them into vascular segments of increasing diameters.

As evident from On-line Fig 1 and the Table, sizing mismatch invariably leads to substantial heterogeneity in metal coverage and porosity across the aneurysm neck as the device transitions from a constrained diameter to its unconstrained state (the degree of which may be mitigated by deployment technique), even under circumstances of relatively modest oversizing. Although the clinical significance of such mismatch in terms of reduced efficacy or potential deleterious changes in dynamic intra-aneurysmal flow is undefined as yet, sporadic adverse reports of worsening mass effect and delayed aneurysm rupture after treatment of large aneurysms with flow-diversion devices suggest a need for understanding the likely in situ disposition of the devices and the potential effects that suboptimal deployment may have on the hemodynamic condition of the aneurysmal environment. ${ }^{11,12}$

On the other hand, in different circumstances, deliberate oversizing could provide a potential advantage as a means to reduce metal coverage of eloquent perforators arising from the perianeurysmal regions of the parent artery. In this scenario, an oversized device could be selected to bridge the aneurysm neck (minimizing perforator coverage distal to the aneurysm), with additional (shorter) devices deployed across the aneurysm neck to address heterogeneities in the coverage of the aneurysm. This implicit duality with respect to therapeutic intention (maximized cover- age of the aneurysm neck, minimized coverage of eloquent branch vessels) mandates a greater understanding of the device and its behavior under specific conditions, to optimize constructs to best accommodate the unique features of each aneurysm and the adjacent vascular environment.

\section{Strategies to Address Vessel Size Mismatch}

In addressing concerns related to transition zone effects, various strategies using multiple, shorter devices may minimize such effects (Fig 3). One approach exploits the partial overlapping of tandem devices, each appropriately sized for its respective landing zone, with successively larger devices sequentially telescoped to create a variable diameter construct, bridging the overall gap in size. While it is impossible to eliminate all transition effects, the transitions can be made more gradual, decreasing the abruptness of the step-off and shifting the ultimate TZ proximal to the aneurysm neck. This latter benefit can be obtained by extending a distally placed device, appropriately sized to the distal smaller diameter landing zone, fully across the aneurysm neck and, subsequently, anchoring this device within the larger parent artery proximal to the aneurysm with a second device, selected to match the larger diameter landing zone. The effective consequence of this construct is to move the $\mathrm{TZ}$ proximal to the aneurysm where the larger device emerges from the constraint imposed by the smaller diameter PED. This approach also provides higher metal coverage in the region of device overlap, which typically falls across the aneurysm neck (Fig 7). Alternatively, to address a perforator-rich territory (P1 segment, M1 segment) distal to an aneurysm, a construct can be built from proximal to distal, using a device sized to the vessel proximal to the aneurysm to cover the aneurysm neck, followed by a larger device, oversized to the smaller diameter vascular segment distal to the aneurysm and constrained by the proximal device across the aneurysm neck-thereby, potentially reducing the metallic coverage throughout the perforator-rich distal segment and providing double coverage of the aneurysm neck.

Although coverage initially decreases sharply with oversizing, due to the parabolic relationship between porosity and device diameter, surface coverage (in straight vascular segments) after reaching minimum values (near 20\% for PED; On-line Fig 1, Table) can be expected to sharply increase at further constrained diameters. For example, the $4.75-\mathrm{mm}$ device provides a minimum coverage of $18 \%$ when constrained within tubes ranging from 3 to $4 \mathrm{~mm}$ (Table), compared with a maximum of $27 \%$ when 

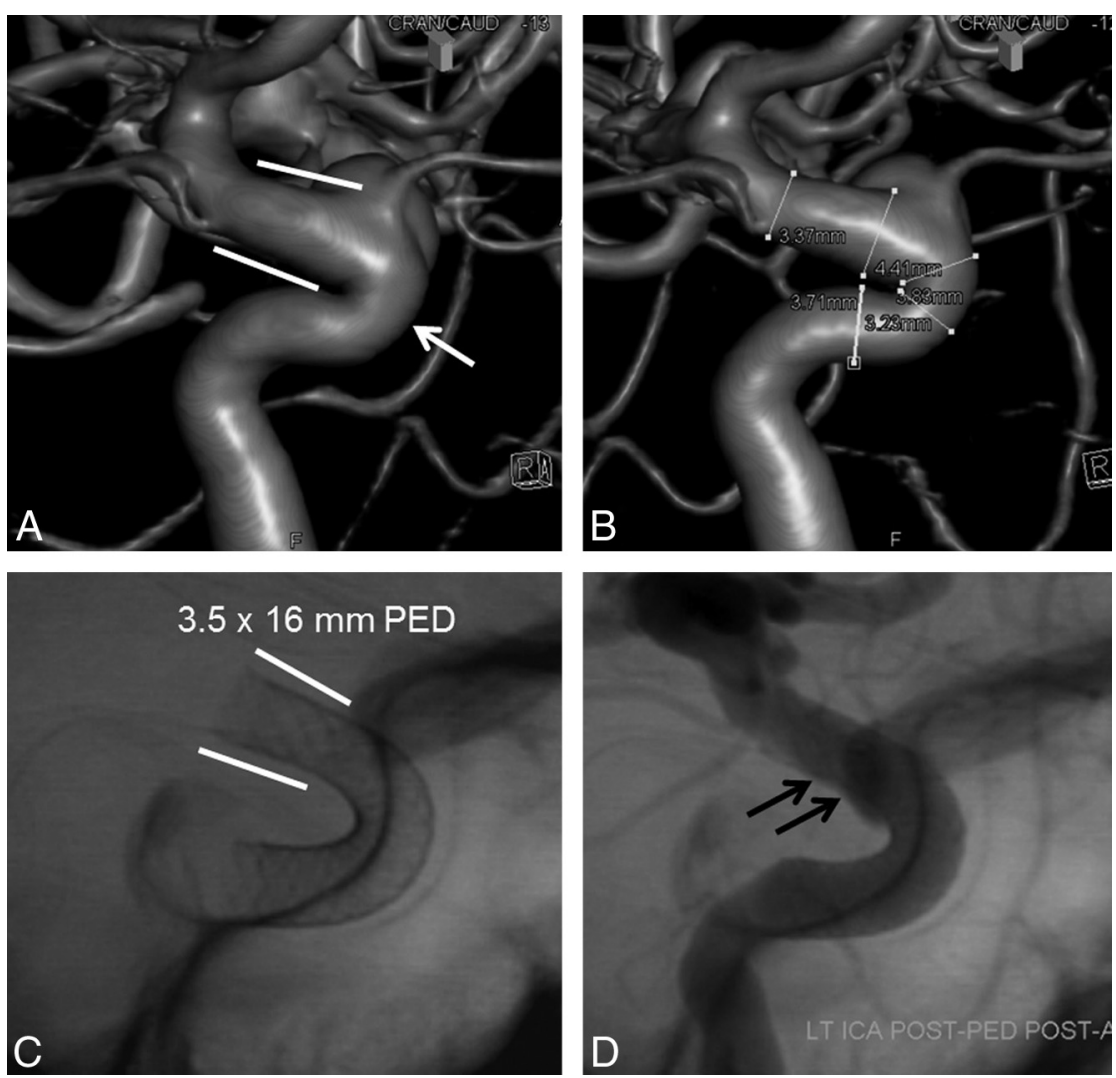

FIG 5. In vivo illustration of the memory shape effects. $A$ and $B$, A wide-neck ophthalmic segment aneurysm with associated ectasia of the parent vessel, which tapers down from the ophthalmic artery to the ostium of the posterior communicating artery (paired white lines). The carotid artery is, however, normal in caliber at the anterior genu (white arrow). C and D, Native and native + contrast lateral views following Pipeline deployment. Notice the reverse morphology of the devices with the maximal diameter at the distal end ( $C$, paired white lines). Although the device was deployed with an appropriate load, the memory effect at the anterior genu resulted in incomplete opening of the device at the level of the ophthalmic artery ( $D$, black arrows). It is necessary to make sure that the distal end is fully in contact with the vessel to prevent an endoleak in this scenario.

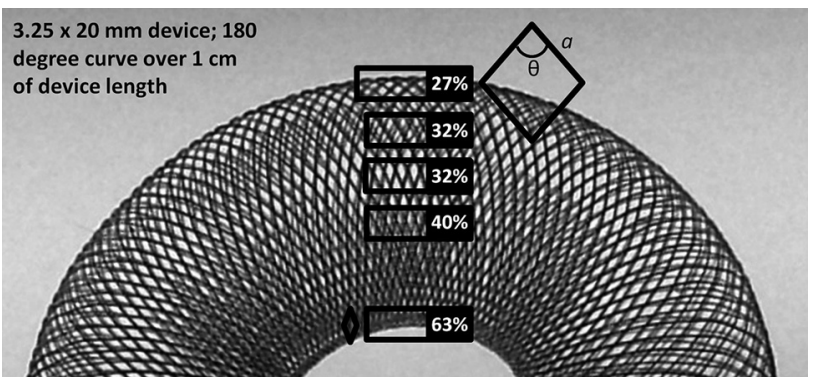

FIG 6. Photograph of a $3.25 \times 20 \mathrm{~mm}$ device along a $180^{\circ}$ curvature taking up $\sim 10 \mathrm{~mm}$ of the device. The percentage of metal coverage at each of the stations along the curve is listed, with a corresponding illustration of cell shape, showing that both $\theta$ and cell side length $a$ vary substantially along the curve.

deployed in a 5.0-mm tube. Below $2.75 \mathrm{~mm}$, coverage again significantly increases. An implication of the observed coverage minimae measured for PEDs of differing diameters is that doublecovering a segment of low coverage with a device of identical or larger diameter (to take advantage of differences in deployed pitch between overlapped devices) will increase coverage to $>30 \%$, which would be superior to the percentage coverage with a single device at its nominal opening state.
Also important is the recognition that the surface coverage of a device is fundamentally related to its deployed diameter; furthermore, in its unconstrained state, slight increases in diameter occurring with longitudinal compression of the device (loading) can drive coverage (along its parabolic curve) to very high levels. Judicious "packing" of the device is important to assure its optimal apposition to the recipient vessel. However, beyond this, for device segments optimally apposed to the vessel wall and, therefore, constrained by the parent artery, no increase in coverage within the vessel can be achieved by applying forward load onto the device. This may limit packing of the device across aneurysms with small necks. In contrast, packing (by loading the device during deployment) can substantially increase coverage in the unconstrained portion of the PED, across the aneurysm neck or within a fusiform aneurysmal segment-bearing in mind that, much like landing-zone coverage, the limited TZ coverage is not ameliorated by this strategy.

Theoretically, TZ effects could be reduced by controlling the deployed diameter of the device across the aneurysm neck by unsheathing the device under slight traction, rather than pushing it out under load. However, this must be done carefully, ensuring that the device becomes sufficiently implanted proximal to the aneurysm (possibly supported by additional anchoring devices) to avoid subsequent "migration" of the unloaded "stretched" bridging device by preventing potential gradual self-expansion to its nominal diameter in the postprocedural phase.

\section{Curve-Related Porosity Changes}

The relationships between device diameter and metal coverage in this study were calculated for devices deployed in linear models. The degree of metallic coverage observed in vivo, however, will be substantially more complex due to the effects of vessel curvature. In curved vascular segments, the constituent filaments of the device slide over one another, altering not only the angles of the rhomboids but also their side-length dimensions (Fig 6), giving rise to a variable "porosity" at each point along the vessel crosssection, from the inner to the outer curves. From a clinical standpoint, the reduced coverage (higher porosity) along the outer curve may become important in the treatment of outer curvature aneurysms, possibly requiring additional devices to achieve a therapeutic degree of coverage across the aneurysm neck, as previously observed by Darsaut and colleagues, ${ }^{13}$ both in vitro and in animal models. ${ }^{8}$ Conversely, there should be heightened concern 


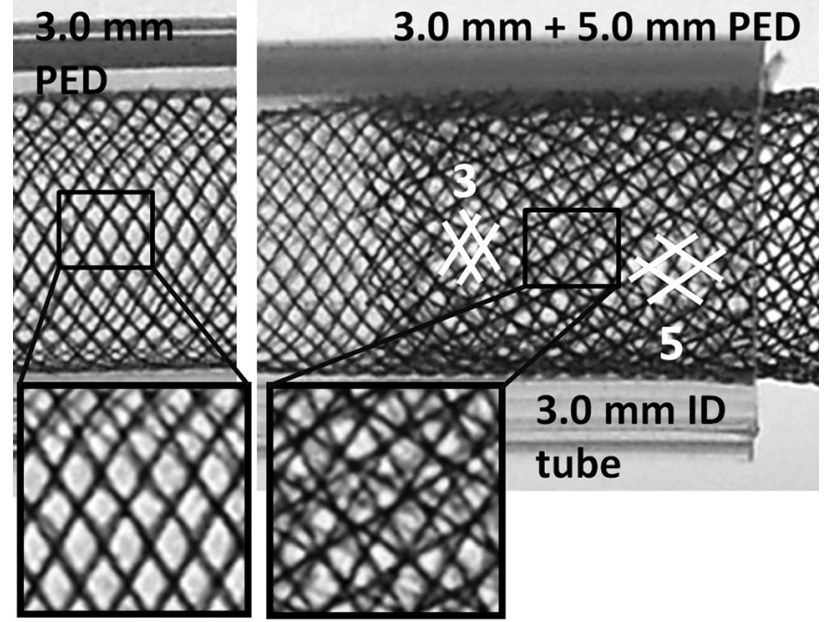

FIG 7. Illustration of double-coverage effects on the extent and morphology of metal coverage. High-magnification views of the cell structure of a single $3.0-\mathrm{mm}$ device deployed in a $3.0-\mathrm{mm}$ tube; a $5.0-\mathrm{mm}$ device is then telescoped into the $3.0-\mathrm{mm}$ device. Note the much larger cell size of the $5-\mathrm{mm}$ device and different angles of overlapping pitch from each device.

for perforators originating from the inner curvature, where coverage values increase to very high numbers. There are some in vitro data to support the notion of increased neointimal overgrowth across side-branch ostia with $>35 \%-40 \%$ metal coverage. $^{14,15}$

The results of our experiments with the PED may be only qualitatively applicable to other braided flow-diverter devices. For example, a detailed study of the Silk device (Balt Extrusion, Montmorency, France) by Aurboonyawat et $\mathrm{al}^{5}$ showed that this device behaves quite differently with respect to oversizing and curvature, a difference that seems to be related to the variability in nominal pitch angle $\theta$ between the 2 devices.

Given the many configurations that vascular segments (each exhibiting distinct stenoses and complex curvatures) and devices implanted within them may assume, the extent to which in vitro observations can be applied to realistic in vivo scenarios remains an open question. Nevertheless, the results presented here serve to illustrate the importance of understanding fundamental characteristics of the devices and critical anatomic features (changing vessel diameters, the nature of the aneurysm neck, the curvature of the target vascular segment, stenoses) among the number of factors that influence case selection and treatment strategy.

\section{CONCLUSIONS}

We present benchtop observations documenting important geometric properties of the Pipeline Embolization Device in various scenarios. Knowledge of these device geometries should be helpful to operators using the device, particularly under the challenging anatomic circumstances often necessitating its use. Our findings quantitatively illustrate that device metal coverage is a dynamic value with substantial variability under realistically expected deployment conditions. We hold these results as support- ive of the need to consider regional metal coverage in devicetreatment strategies, which may require the use of multiple devices to achieve the desired geometric configurations and maximize the overall efficacy of treatment.

Disclosures: Maksim Shapiro-UNRELATED: Consultancy: Covidien, Comments: Pipeline proctor consultant with Covidien, Payment for Lectures (including service on Speakers Bureaus): Covidien, Comments: participates in Pipeline physician training courses with Covidien, Payment for Development of Educational Presentations: Covidien, Comments: developed Pipeline educational presentations for physician and staff training with Covidien. Tibor Becske-UNRELATED: Consultancy: ev3/ Covidien, Payment for Development of Educational Presentations: ev3/Covidien, Comments: payment for development of Pipeline training materials, Travel/Accommodations/Meeting Expenses Unrelated to Activities Listed: ev3/Covidien, Comments: travel and accommodation support, Other: ev3/Covidien, Comments: proctoring fees. Peter K. Nelson—UNRELATED: Consultancy: Covidien, Comments: fees covering physician proctoring.

\section{REFERENCES}

1. Nelson PK, Lylyk P, Szikora I, et al. The Pipeline embolization device for the Intracranial Treatment of Aneurysms trial. AJNR Am J Neuroradiol 2011;32:34-40

2. Becske T, Kallmes DF, Saatci I, et al. Pipeline for uncoilable or failed aneurysms: results from a multicenter clinical trial. Radiology 2013;267:858-68

3. Wang K, Yuan S. Actual metal coverage at the neck is critical for flow-diverting stents in treating intracranial aneurysms. AJNR Am J Neuroradiol 2013;34:E31-32

4. Makoyeva A, Bing F, Darsaut TE, et al. The varying porosity of braided self-expanding stents and flow diverters: an experimental study. AJNR Am J Neuroradiol 2013;34:596-602

5. Aurboonyawat T, Blanc R, Schmidt P, et al. An in vitro study of Silk stent morphology. Neuroradiology 2011;53:659-67

6. Mut F, Cebral JR. Effects of flow-diverting device oversizing on hemodynamics alteration in cerebral aneurysms. AJNR Am J Neuroradiol 2012;33:2010-16

7. Wang K, Huang Q, Hong B, et al. Correlation of aneurysm occlusion with actual metal coverage at neck after implantation of flow-diverting stent in rabbit models. Neuroradiology 2012;54:607-13

8. Bing F, Darsaut TE, Salazkin I, et al. Stents and flow diverters in the treatment of aneurysms: device deformation in vivo may alter porosity and impact efficacy. Neuroradiology 2013;55:85-92

9. Darsaut TE, Bing F, Salazkin I, et al. Testing flow diverters in giant fusiform aneurysms: a new experimental model can show leaks responsible for failures. AJNR Am J Neuroradiol 2011;32:2175-79

10. Darsaut TE, Bing F, Salazkin I, et al. Flow diverters can occlude aneurysms and preserve arterial branches: a new experimental model. AJNR Am J Neuroradiol 2012;33:2004-09

11. Kulcsár Z, Szikora I. The ESMINT Retrospective Analysis of Delayed Aneurysm Ruptures after flow diversion (RADAR) study. In: Proceedings of the Annual Meeting of the European Society of Minimally Invasive Neurological Therapy, September 2012, Nice, France

12. Kulcsár Z, Houdart E, Bonafé A, et al. Intra-aneurysmal thrombosis as a possible cause of delayed aneurysm rupture after flow-diversion treatment. AJNR Am J Neuroradiol 2011;32:20-25

13. Darsaut TE, Bing F, Salazkin I, et al. Flow diverters failing to occlude experimental bifurcation or curved sidewall aneurysms: an in vivo study in canines. J Neurosurg 2012;117:37-44

14. Hong B, Wang K, Huang Q, et al. Effects of metal coverage rate of flow diversion device on neointimal growth at side branch ostium and stented artery: an animal experiment in rabbit abdominal aorta. Neuroradiology 2012;54:849-55

15. Lieber BB, Sadasivan C. Endoluminal scaffolds for vascular reconstruction and exclusion of aneurysms from the cerebral circulation. Stroke 2010;41:S21-25 